\title{
A Study of Factors Influencing Plant Growth by WSN Approach and Plant Nutrient Deficiency Classification in Tomato Using SVM
}

\author{
Vrunda Kusanur \\ Department of Electronics and Communication Engineering \\ BNM Institute of Technology, Bengaluru, Karnataka, India \\ Email-vvrundakusanur@bnmit.in \\ Veena S Chakravarthi \\ Department of Electronics and Communication Engineering \\ BNM Institute of Technology, Bengaluru, Karnataka, India \\ Email-veenasc@bnmit.in
}

\begin{abstract}
Soil temperature and humidity straight away influence plant growth and the availability of plant nutrients. In this work, we carried out experiments to identify the relationship between climatic parameters and plant nutrients. When the relative humidity was very high, deficiency symptoms were shown on plant leaves and fruits. But, recognizing and managing these plant nutrients manually would become difficult. However, no much research has been done in this field. The main objective of this research was to propose a machine learning model to manage nutrient deficiencies in the plant. There were two main phases in the proposed research. In the first phase, the humidity, temperature, and soil moisture in the greenhouse environment were collected using WSN and the influence of these parameters on the growth of plants was studied. During experimentation, it was investigated that the transpiration rate decreased significantly and the macronutrient contents in the plant leave decreased when the humidity was $\geq 95 \%$. In the second phase, a machine learning model was developed to identify and classify nutrient deficiency symptoms in a tomato plant. A total of 880 images were collected from Bingo images to form a dataset. Among all these images, $80 \%$ (704 images) of the dataset were used to train the machine learning model and 20\% (176 images) of the dataset were used for testing the model performance. In this study, we selected K-means Clustering for keypoints detection and SVM for classification and prediction of nutrient stress in the plant. SVM using linear kernel performed better with the accuracy rates of $89.77 \%$ as compared to SVM using a polynomial kernel.
\end{abstract}

\section{Keywords: Nutrient deficiency, WSN, SVM, K-means}

\section{INTRODUCTION}

Due to advancements in science and technology, traditional agriculture has been transformed by modern technology. To get a better yield, crops are grown under a controlled and closed environment (greenhouse) to protect the crop from excess temperature, wind, and rainfall. However, there are many challenges for growers to get better in the greenhouse.

According to Richard G. Snyder [1], growing tomatoes in a Greenhouse is not easy because of specific production requirements. In the greenhouse, the temperature will not depress at night and plant leaves will get damp from the rain. Consequently, the roots cannot absorb water and nutrients well. Due to high temperature and relative humidity, insects, and diseases are constant threats. Redmond Ramin Shamshiri et al.[2],mentioned that regular attention is needed for tomatoes grown in the greenhouse environment. For greenhouse farming, the best 
values of air and root-zone temperature, relative humidity, and vapour pressure are summarized for successful greenhouse farming. As per H.R. Gislerod et al.[3], the amount of Calcium decreases with a decrease in temperature. 3 Authors observed the influence of air humidity on nutrients using nine different greenhouse species. A proper nutrient solution is provided once every two weeks for every species. There was a remarkable reduction in transpiration rate when relative humidity $(\mathrm{RH})$ was raised from a smaller value to a larger value. According to the authors, the content of macronutrient elements in the plant leaves is reduced by rising relative humidity. This reduction was serious only for Potassium and Calcium in Lycopersicon and KjeldahlN in Nephrolepis. At the end of the experiment, when the plants have been growing at high $\mathrm{RH}$, the content of the macronutrient elements in the growth medium was the smallest. Nitrogen and Potassium were the most affected components.

Margit Olle et al.[4], experimented in their research work that the problems that occur with tomatoes are not only caused by insects or diseases but also due to environmental parameters such as temperature, humidity, light, water, or nutrition and are termed as "physiological disorders". Blossom end rot (BER) and Fruit Cracking (FC) are the two dominant physiological disorders that can damage Tomato. Physiological Ca deficiency is generally correlated to the failure of the plant to transfer sufficient Calcium to the affected plant part, rather than inadequate Calcium levels in the growing medium. During the time the fruit starts to ripen, a small darkened or water-soaked area around the blossom end of the fruit appears and is the first visible symptom of BER. As the fruit matures, the place darkens, expands, and becomes sunken. FC takes place when there is a quick net influx of solutes and water into the fruit. The fruit ripens prematurely due to this Blossom-End Rot. Temperature affects all the phases in plant development (vegetative growth, flowering, fruit seting, and fruit ripening).

D. Devi Kala Rathinam et al. [5] conducted a study on solutions for real-time problems using Wireless Sensor Network (WSN) technology. Recently WSN established widespread applications in several fields like transport, medical, military, mobile phones, home appliances, and so on. Low-cost WSN can be used to monitor, measure climatic change, irrigation system, measuring water supply. Wireless Sensor Nodes are used to monitor temperature, humidity to increase productivity and for smart agriculture with less human effort. The collected data is stored in the cloud or memory. Dattatraya Shinde and Naseem Siddiqui[6] designed a simple circuit to calibrate and control the greenhouse environment parameters using Raspberry Pi 3. WSN is used to measure the quality of soil to find out whether the field is dry or wet. Two crops chosen are Tomato and Brinjal for the prediction and analysis process in this research. Artificial Intelligence (AI) finds its application to resolve numerous issues and to improve the production and performance processes in the agriculture, food, and bio-system engineering fields. Kefaya Qaddoum et al.[7] collected the temperature, CO2, Vapour-pressure deficit (VPD), radiation, and past yield for yield prediction during the period 2004 to 2008 using the "Evolving Fuzzy Neural Network" $(\mathrm{EFuNN})$ model. The weekly variations in the crop yield are predicted with an average accuracy of $90 \%$ by EFuNN model. The authors also correlated the accuracy of EFuNN model with the Bayesian model and RBF networks. Knowing the information in advance would help the growers to make decisions on climate and to manage the crop. Sulistyo S.B. et al.[8], estimated the nitrogen content in wheat leaves using combinations of backpropagation neural networks. A digital camera was used to catch the leaf images under sunlight. The input parameters used are features of three color spaces i.e. RGB, HIS, and CIE-Lab to predict the nitrogen content. The leaves are differentiated from other surrounding parts using a neural network in the image segmentation. Better results were observed by authors as 
compared with linear regression analysis and single neural network-based estimation methods.

From all the above studies, we observe that temperature, humidity, light, etc. are the important parameters and must be monitored and controlled for plants in the greenhouse environment. The influence of relative humidity on macronutrient elements was focused on. The problems that occur with tomatoes are investigated in the greenhouse are not only due to pests and insects but also due to the greenhouse environment and nutrients. Tomato is damaged by two critical physiological disorders-Blossom End Root (BER) and Fruit Cracking (FC). The different ways to diagnose the nutrient deficiencies are discussed and visual observation is an effective and fastest method to identify plant nutrients in the crops. The research on applications of cost-effective WSN in greenhouse automation for smart agriculture and storing the gathered data on the cloud or in memory was conducted.

\section{Objective}

As mentioned above, extensive research was done on different kinds of diseases in plants and yield prediction to increase the crop yield in the feature using various deep learning architectures. Evaluating the performance of machine learning algorithms to classify predict calcium and magnesium deficiencies for tomato crops is the primary objective of this research.

The proposed work is organized as follows: Section two shows the suggested WSN model to monitor the temperature, humidity of the soil and investigate the effect of these environmental parameters on plant nutrients. Section three shows the dataset collection with calcium and magnesium deficiencies and prediction of plant nutrients using Support Vector Machine (SVM). In section four, the results of the k-means algorithm and SVM approach for the classification and prediction of plant nutrients are discussed. In section five, the conclusion of the proposed paper is given.

\section{Proposed Method}

The overview of the proposed architecture is shown in figure 1. It comprises of two models: One is the Wireless Sensor Network model for Climate monitoring and the other one is the Machine Learning model for nutrient deficiency detection.

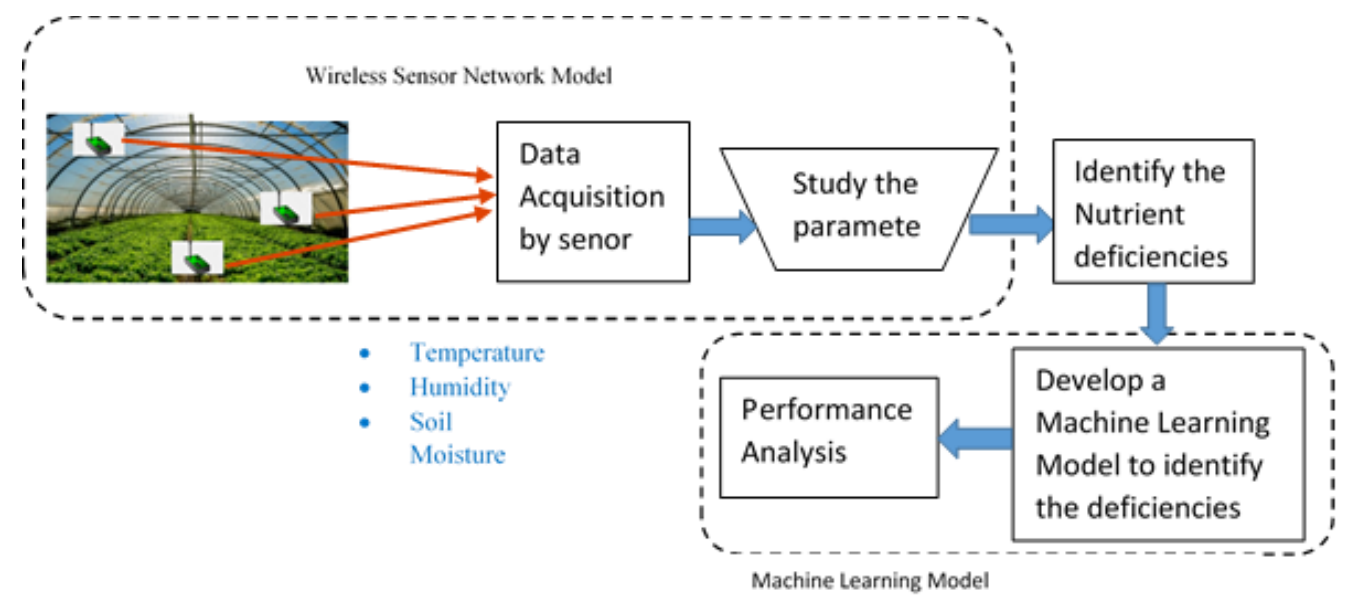

Figure 1. Overview of Proposed System 


\subsection{Wireless Sensor Network for Climate Monitoring}

To understand the effects of temperature, humidity, and moisture on plant growth, it is necessary to observe the greenhouse environment to determine and control the environmental parameters.

Totally three sensor nodes are used where each one is capable of sensing temperature, humidity, and soil moisture. The information gathered from sensor node- 1 and node- 2 are transmitted to node-3 through wireless communication. Here, Sensor node-3 behaves as an administrator node and behaves as a gateway between Zigbee and GSM. All the sensor node includes sensor unit and computing system. The sensor measures the data from an environment and sends it to a microprocessor (PIC16F877A) which processes and transmits the data using a transceiver.

3.1.1. Temperature and Humidity Sensor (SHT71): SHT71 sensor detects ambient temperature and humidity through the standard single-wire interface. It provides the digital output and has an accuracy of $\pm 0.5^{\circ} \mathrm{C}$ for temperature \& $0.2 \%$ for humidity. Humidity is the amount of water in the form of vapour in a body of air. The humidity is measured by several methods. One of the most usual methods to measure humidity is using Relative Humidity $(\mathrm{RH})$. At the given temperature and pressure, the ratio of the quantity of water vapour corresponding to the maximum water vapour that the air can hold at a given temperature and pressure is defined as the relative humidity $(\mathrm{RH})$. It is defined by (1) as follows.

$\mathrm{RH} \%=\frac{P_{v}}{P_{s}} \times 100$

Where $\mathrm{Pv}$ is the partial pressure of water vapour in air and Ps is the saturated water vapour pressure of air at the same temperature. The surrounding air is measured by using a capacitive humidity sensor and a thermistor.

3.1.2. Soil Moisture Sensor (YL-69): YL-69 sensor is used to detect the humidity of the soil. To adjust the sensitivity of the digital output (D0), the built-in potentiometer of the sensor is used. The sensor output voltage changes according to the water content in the soil. When the soil is wet, the output voltage decreases and it increases when the soil is dry. D0 is the digital output signal from the module and it will be LOW if the soil humidity exceeds a threshold value otherwise it will be HIGH. It also supports an analog output signal from 0 to 1023.

3.1.3. Microcontroller (PIC16F877A): The microcontroller PIC16F877A is used as the computing device. It has a total of 40 pins out of which 33 pins are for input and output operation. It has 35 instructions in the instruction set. It operates up to $20 \mathrm{MHz}$ frequency.

3.1.4. Data-Acquisition: The sample data including ambient temperature, humidity, and soil moisture gathered from one of the three sensor nodes is shown in table 1. From the sensor data, it was observed that on 24-04-2017 from 17:00:00 to 23:00:00 when the humidity was $95 \%$, the transpiration rate decreased significantly and the macronutrient contents in the plant leaves decreased. Nitrogen, Calcium, Magnesium, and Potassium were the mainly affected elements.

Section 3.2 presents the Machine learning model and data collection with calcium and magnesium deficiencies in the tomato plant. 
Table 1. Data Gathered from Sensor Node-2

\begin{tabular}{|c|c|c|c|c|c|c|}
\hline \multicolumn{7}{|c|}{ SENSOR DATA FROM SENSOR NODE-2 } \\
\hline DATE & Time & $\begin{array}{c}\text { Amb_Temp } \\
\left({ }^{\circ} \mathbf{C}\right)\end{array}$ & $\begin{array}{c}\text { Humidity } \\
(\%)\end{array}$ & $\begin{array}{c}\text { Soil } \\
\text { Moisture }\end{array}$ & Lat & Lon \\
\hline 15-02-2017 & 07:00:00 & 17 & 62 & 765 & 12.96 & 77.6414 \\
\hline 15-02-2017 & 13:00:00 & 27 & 27 & 765 & 12.96 & 77.6414 \\
\hline 15-02-2017 & 19:00:00 & 20 & 53 & 765 & 12.96 & 77.6414 \\
\hline 16-02-2017 & 07:00:00 & 15 & 54 & 765 & 12.96 & 77.6414 \\
\hline 16-02-2017 & 13:00:00 & 26 & 29 & 765 & 12.96 & 77.6453 \\
\hline 16-02-2017 & 19:00:00 & 19 & 48 & 765 & 12.96 & 77.6453 \\
\hline 17-02-2017 & 07:00:00 & 15 & 62 & 760 & 12.96 & 77.6453 \\
\hline 17-02-2017 & 13:00:00 & 26 & 33 & 750 & 12.96 & 77.6453 \\
\hline 17-02-2017 & 19:00:00 & 20 & 53 & 755 & 12.96 & 77.6453 \\
\hline 18-02-2017 & 07:00:00 & 16 & 52 & 755 & 12.96 & 77.6453 \\
\hline 18-02-2017 & 13:00:00 & 29 & 24 & 755 & 12.96 & 77.6453 \\
\hline 18-02-2017 & 19:00:00 & 21 & 50 & 755 & 12.96 & 77.6453 \\
\hline • & • & • & • & • & - & - \\
\hline$\cdot$ & • & - & - & • & - & • \\
\hline 20-04-2017 & 13:00:00 & 28 & 47 & 1017 & 12.96 & 77.6453 \\
\hline 20-04-2017 & 19:00:00 & 24 & 71 & 1017 & 12.96 & 77.6453 \\
\hline 21-04-2017 & 07:00:00 & 29 & 76 & 1017 & 12.96 & 77.6453 \\
\hline 21-04-2017 & 13:00:00 & 30 & 95 & 1017 & 12.96 & 77.6453 \\
\hline 21-04-2017 & 19:00:00 & 30 & 95 & 1017 & 12.96 & 77.6453 \\
\hline 22-04-2017 & 07:00:00 & 30 & 95 & 1017 & 12.96 & 77.6453 \\
\hline 22-04-2017 & 13:00:00 & 30 & 95 & 1017 & 12.96 & 77.6453 \\
\hline 22-04-2017 & 19:00:00 & 30 & 95 & 1017 & 12.96 & 77.6453 \\
\hline
\end{tabular}

\subsection{Machine Learning Model for Nutrient Deficiency Ddetection}

The machine learning model shown in figure 2 to detect nutrient deficiencies has 6 steps.

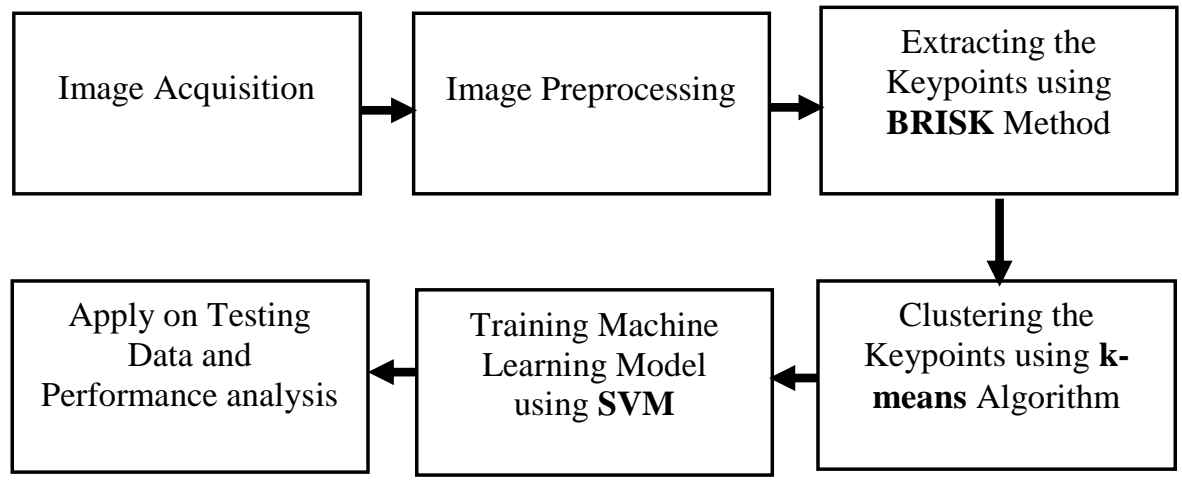

Figure 2. Machine Learning Model for Nutrient deficiency detection

3.2.1. Image Acquisition: In the image acquisition step, the tomato leaves and fruit dataset is collected from google images and bingo images for training and testing. In the proposed work, the dataset has two nutrient deficiencies i.e. Calcium and Magnesium for classification purposes. There are 880 images which are divided into two parts- 80\% (704 images) of the dataset are used to train the machine learning model and 20\% (176 images) of the dataset is used for testing the model performance. Out of 704 training images, 374 images are of Calcium deficiency and the remaining 330 are of Magnesium deficiency. Out of 176 testing images, 94 images are of Calcium deficiency and 82 images are of Magnesium deficiency. 
3.2.2. Image Preprocessing: Noise or the other object removal was done in the Image Preprocessing step. Also, all the images were resized to 255 X 255 during.

3.2.3. Keypoints Extraction: Keypoints are points of interest in an image that can be used to compare images and perform tasks such as image alignment and registration. There are many popular algorithms such as SIFT, SURF, BRISK, and ORB to detect these keypoints automatically. Along with detectors, these algorithms also define descriptors that are useful for image processing tasks. In this work, Binary Robust Invariant Scalable Keypoints (BRISK) method is used for extracting the Keypoints and descriptors. There are three stages in the BRISK method: Keypoint detection, Keypoint description, and Keypoint matching.

3.2.3.1. Keypoint Detection Stage: In the feature detection stage, the accurate range of each keypoint in the continuous scale-space is evaluated by the BRISK detector. There are $\mathrm{n}$ octaves ci and $\mathrm{n}$ intra-octaves di in scale-space pyramid layers of BRISK framework for $\mathrm{i}=$ $\{0,1 \ldots \ldots n-1\}$. The typical value of $n$ is 4 . The octaves are formed by continuously halfsampling an actual image (corresponding to $\mathrm{c} 0$ ). Each intra-octave di is situated in the middle of layers ci and ci+1. The first intra-octave $\mathrm{d} 0$ is computed by downsampling an actual image $\mathrm{c} 0$ by 1.5 . By consecutively sampling by half, the leftover intra-octave layers are derived as shown in (2)

$$
\begin{aligned}
& \mathrm{t}\left(\mathrm{c}_{\mathrm{i}}\right)=2^{\mathrm{i}} \\
& \mathrm{t}\left(\mathrm{d}_{\mathrm{i}}\right)=2^{\mathrm{i}} .1 .5
\end{aligned}
$$

3.2.3.2. Keypoint Descriptor Stage: The following are the six steps in keypoint description.

1. Sample pattern of soft pixels all over the characteristic.

2. Divide a couple of pixels into two subgroups: short-distance pairs, and long-distance pairs.

3. Compute the local gradient in the middle of long-distance pairs.

4. Determine feature orientation by adding gradients.

5. Solve short-distance pairs using orientation.

6. Develop a binary descriptor from rotated short-distance pairs.

3.2.3.3. Keypoint Matching Stage: Resemblance amongst the descriptors of pair of feature points has to be compared to perform the descriptors matching. The extracted feature points are expressed in the bitstream ( 1 and 0$)$. To find the resemblance amongst images, Hmming distance has to be determined for all pairs between images (Two 512 bit strings) and is implemented using a bitwise XOR operation. By finding the value of Hamming distance, the degree of two BRISK descriptors matching is evaluated. The degree of descriptors matching reduces as Hamming distance increases.

3.2.4. Keypoints Clustering using K-Means algorithm: K-Means is a simple and fast algorithm. It is a popular classification algorithm that partitions the objects into k groups or clusters based on their features. The objective of K-Means algorithm is to reduce the squared Euclidean length in the middle of the feature point and the centroid of a belonging cluster. There are three steps in the algorithm.

1. Initialization: $\mathrm{k}$ centroids are formed after choosing the number of group $\mathrm{k}$. 
2. Designation of substance to the centroids: Designating the observation one by one to its neighboring centroid.

3. Centroids reform: Pick the new centroid the average positions of the observation to which it belongs to reform the location of the centroid of each cluster.

An optimization problem would be resolved by the k-means algorithm. An optimized function is the summation of the quadratic distance from all the observations to its centroid of a cluster. The observation is expressed by vectors $(\mathrm{x} 1, \mathrm{x} 2, \ldots, \mathrm{xn})$ with dimension $\mathrm{d}$. $\mathrm{k}$ groups are constructed and the summation of the distances of the observations to its centroid is reduced within each group $\mathrm{S}=\{\mathrm{S} 1, \mathrm{~S} 2, \ldots, \mathrm{Sk}\}$ using the k-Means algorithm. The problem can be formulated as shown in (3)

$\min _{s} E(\mu i)=\min _{s} \sum_{i=1}^{k} \sum_{x j \in S i}\|x j-\mu i\| l 2$

Where $S$ represents the dataset. The members of $S$ are the observations $x j$ indicated by vectors. Every member in $\mathrm{S}$ defines a characteristic. ${ }^{\mu i}$ is the centroid for $\mathrm{k}$ clusters.

$\frac{\partial E}{\partial \mu i}=0 \Rightarrow \mu i(t+1)=\frac{1}{|S i(t)|} \sum_{x j \in S i(t)} x j$

3.2.5. Model Training and classification using SVM: There are many classification algorithms to classify and predict the given input for a classification problem. Popular among all these are Logistic Regression, Support Vector Machine, Decision Trees, k-Nearest Neighbors, and Naïve Bayes algorithms. This work uses the Support Vector Machine (SVM) model to train and predict Calcium and Magnesium nutrient deficiency. The input to SVM is training dataset feature vectors and their related classes, while the classification finding is the output of the model that decides the input image category (Calcium or Magnesium).

For classification and regression problems, SVM is used that determines the ideal hyperplane to distribute the data points between different categories accurately. As much as possible, to avoid errors, the hyperplane is located away from the data. The Kernel Trick technique is applied when the data is not linearly separable. The higher dimensional space would be created using different types of Kernels. Common Kernels used with SVM are Radial Basis Function (RBF), Polynomial, Linear, and Sigmoid. Different types of kernels make different hyperplane decision boundaries between the classes. Usually, training datasets are transformed so that a non-linear decision surface is converted to a linear equation by the kernel function in a larger number of dimension spaces. The kernel function returns the internal product in the middle of two points in an appropriate feature space. The standard Kernel Function is given by (5)

$\mathrm{K}(\overline{\mathrm{x}})=1$ if $\|\overline{\mathrm{x}}\| \leq 1$

$\mathrm{K}(\overline{\mathrm{x}})=0$, Otherwise

In the proposed paper, Linear Kernel and Polynomial Kernel are used to train and test the SVM model to obtain good results. Linear Kernel and Polynomial Kernel are defined by (6) and (7) respectively.

$\mathrm{k}(\mathrm{xi}, \mathrm{xi})=\mathrm{xi} \cdot \mathrm{xj}$

$\mathrm{k}(\mathrm{xi}, \mathrm{xj})=(\mathrm{xi} \cdot \mathrm{xj}+1) \mathrm{d}$

Where $\mathrm{d}$ is the degree of the polynomial and $\mathrm{xi}$ and $\mathrm{xj}$ are vectors.

3.2.6. Performance Analysis: The accuracy is computed for various cluster values $k$ by using the formulae given in (8) 
Accuracy $=\frac{\text { Number of Correct output }}{\text { Total Number of image tried }} X 100$

After the training and classification process, to assess the nutrient deficiency classification model, accuracy and precision are used as the design metrics. To evaluate the performance of the model, the Confusion Matrix is used which describes the performance of the entire model.

\section{Experiment and Result}

The images in the test or validation dataset are used to evaluate the performance of the model. Totally 176 images are used for validation purposes. Out of 176 test images, 94 images are of Calcium deficiency symptoms and the remaining 82 images are of Magnesium deficiency symptoms. Figure 3 shows the accuracy results for different values of $k$ in k-means clustering.

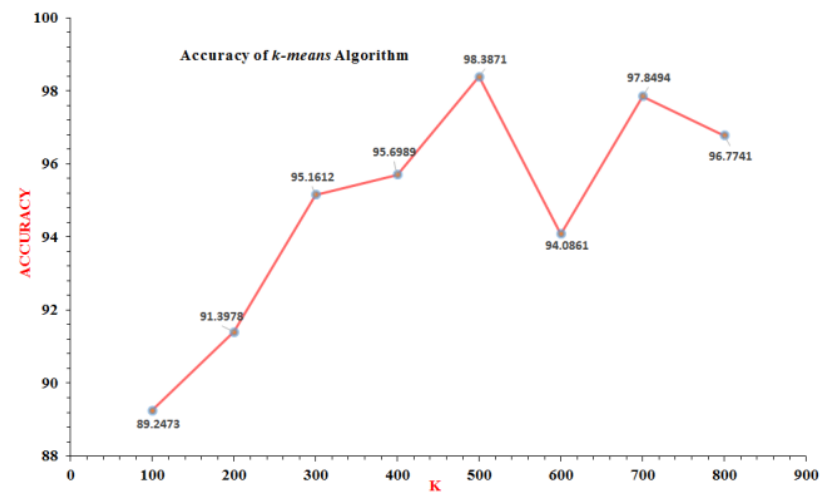

Figure 3. Accuracy of K-Means Algorithm

From figure 3, it is clear that accuracy is increasing as the number of clusters increases in a $\mathrm{K}$-means algorithm. However, after $\mathrm{k}=500$, regardless of an increase in $\mathrm{k}$ value, accuracy is reducing. The results of SVM using Linear Kernel are shown in figure 4. Figure 4A-C shows the results of Calcium deficiency prediction and the forecasting results for Magnesium deficiency are presented in figure 4D-F. The regularization parameter $\mathrm{C}$ is set to 1 and the gamma value is set as auto.

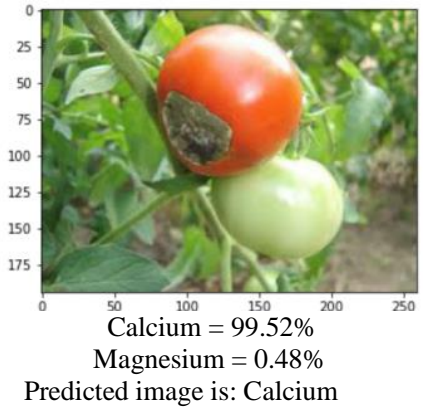

(A)

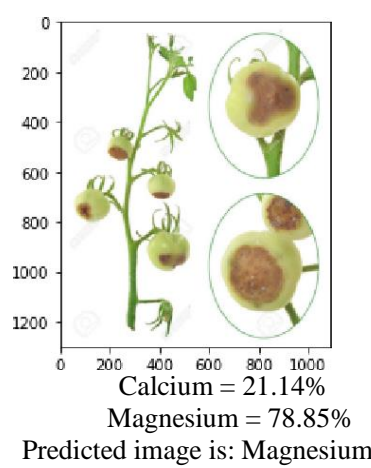

(B)

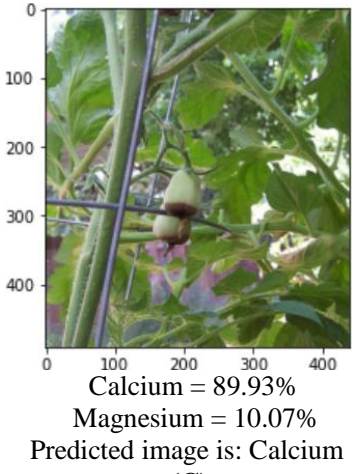

(C) 


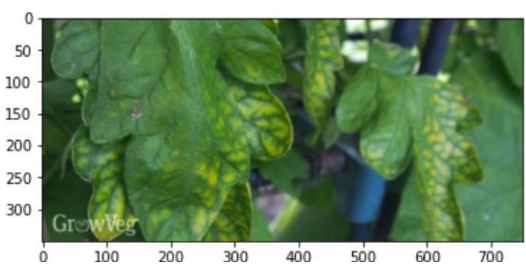

Calcium $=4.54 \%$

Magnesium $=95.46 \%$ Predicted image is: Magnesium

(D)

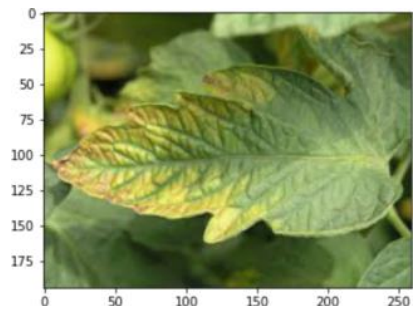

Calcium $=89.03 \%$ Magnesium $=10.97 \%$ Predicted image is: Calcium (E)

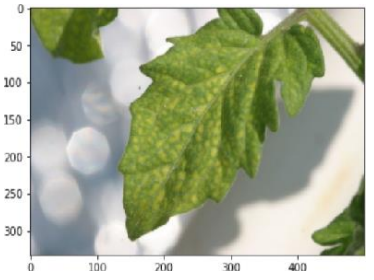

Calcium $=96.23 \%$

Magnesium $=3.77 \%$

Predicted image is: Calcium

(F)

Figure 4. Prediction results of SVM model using Linear Kernel. (A-C) Prediction of Ca deficiency; (D-F) Prediction of Mg deficiency

For the SVM model with the polynomial kernel, the results are as shown in figure 5. Figure $5 \mathrm{~A}-\mathrm{C}$ shows the results of Calcium deficiency prediction and figure 5D-F shows forecasting results of Magnesium deficiency. Regularization parameter $\mathrm{C}$ is set to 1 and the value of the polynomial degree is set to 8 to achieve good results.

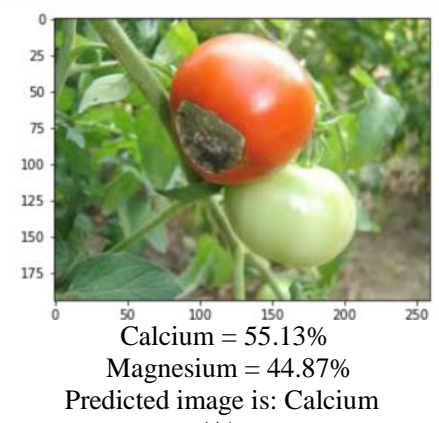

(A)

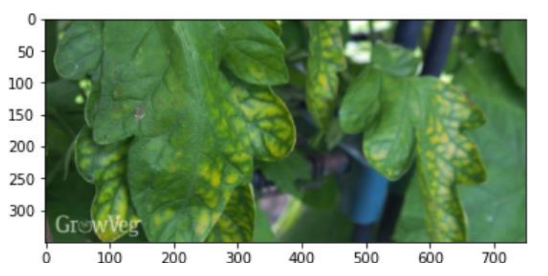

Calcium $=48.00 \%$

Magnesium $=52.00 \%$ Predicted image is: Magnesium

(D)

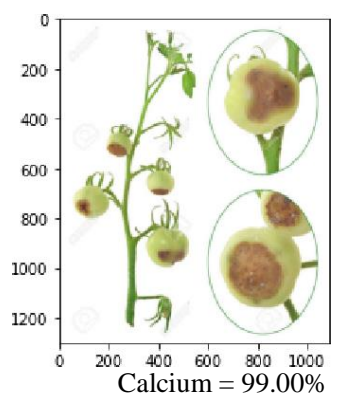

Magnesium $=1.00 \%$

Predicted image is: Calcium

(B)

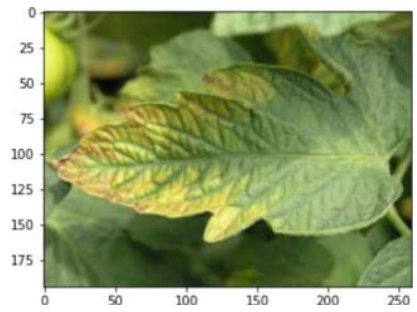

Calcium $=47.35 \%$

Magnesium $=52.65 \%$

Predicted image is: Magnesium

(E)

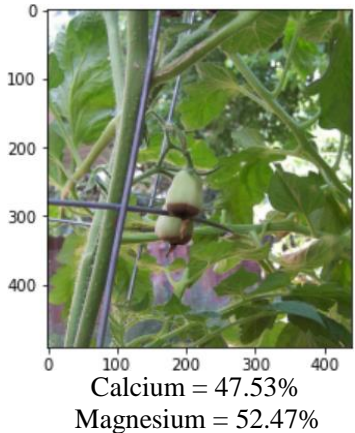

Predicted image is: Magnesium (C)

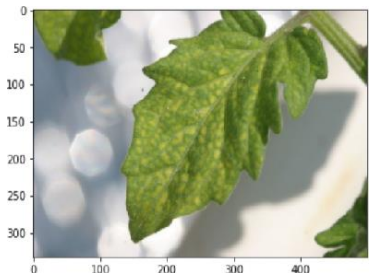

Calcium $=97.28 \%$

Magnesium $=2.72 \%$

Predicted image is: Calcium (F)

Figure 5. Prediction results of SVM model using Polynomial Kernel. (A-C) Prediction of Ca deficiency; (D-F) Prediction of Mg deficiency

The overall accuracy from SVM using linear Kernel is $89.77 \%$ whereas it is $80.68 \%$ from SVM using a polynomial kernel. SVM using linear kernel achieves higher accuracy than SVM using the polynomial kernel in this study. Table 2 and Table 3 present the classifier results for SVM using linear kernel and SVM using polynomial kernel respectively. It can be noticed from tables 2 and 3 that SVM with linear kernel has higher accuracy than SVM with polynomial kernel for the categorization of two nutrients of plants. But, in both models, the accuracy of prediction and classification of $\mathrm{Ca}$ deficiency is less than prediction and classification of $\mathrm{Mg}$ deficiency. 


\section{Conclusion}

Identification of factors influencing the plant nutrients and nutrient deficiency symptoms in the greenhouse environment is a common problem for growers. However, earlier studies only targeted inspection and classification of plant disease. Accordingly, precise evaluation and prediction of the early stages of nutrient deficiency are critical for greenhouse formers. To address this key issue, in this work, first, the influence of greenhouse environmental parameters on plant growth experimented and then the SVM model is trained to identify deficiency symptoms of Calcium and Magnesium in Tomato Plants. The summary based on the results are as follows-

- From the experiments, it was observed that the transpiration rate decreased significantly and the macronutrient contents in the plant leaf decreased when the humidity was $95 \%$.

- For complete processing, $k$-means clustering and SVM algorithms result in large accuracy and consume very little time. For the number of clusters $k=500$, the model accuracy is high. The model is trained for 20,000 iterations to learn the features. The model performance is poor for $k>500$.

The overall accuracy from SVM using linear kernel is $89.77 \%$ whereas it is $80.68 \%$ from SVM using a polynomial kernel. But, in both models, the accuracy of prediction and classification of $\mathrm{Ca}$ deficiency is less than prediction and classification of $\mathrm{Mg}$ deficiency.

The solutions derived in this work are acceptable. In the future, the lack of two or more nutrients should be further investigated using CNN structure. However, the proposed system can be applied to different crops and other deficiency symptom forecasting.

Table 2. Confusion matrix for the prediction of SVM using Linear Kernel with 176 images in the validation dataset

\begin{tabular}{|c|c|c|c|c|}
\hline \multirow[b]{2}{*}{ Actual data } & \multicolumn{2}{|c|}{ Predicted data } & \multirow[b]{2}{*}{ Precision } & \multirow[b]{2}{*}{ Recall } \\
\hline & Calcium & Magnesium & & \\
\hline Calcium & 80 & 14 & $95 \%$ & $85 \%$ \\
\hline Magnesium & 4 & 78 & $85 \%$ & $95 \%$ \\
\hline
\end{tabular}

Table 3. Confusion matrix for the prediction of SVM using Polynomial Kernel with 176 images in the validation dataset

\begin{tabular}{ccccc}
\hline & \multicolumn{2}{c}{ Predicted data } & & \\
\cline { 2 - 3 } Actual data & Calcium & Magnesium & Precision & Recall \\
\hline Calcium & 72 & 22 & $86 \%$ & $77 \%$ \\
Magnesium & 12 & 70 & $76 \%$ & $85 \%$ \\
\hline
\end{tabular}

\section{Acknowledgments}

We extend our sincere gratitude to the Management of the BNM Institute of Technology, Bangalore for furnishing us with all the sources needed and all the support to accomplish this work and the subsequent publications. Also, we are grateful to Visvesvaraya Technological Institute (VTU) for offering us an appropriate platform to do our research. 


\section{REFERENCES}

[1] Richard G. Snyder, "Temperature and Relative Humidity," in Greenhouse Tomato Handbook, Mississippi State University Extension Service, 2001, pp. 1-28.

http://extension.msstate.edu/sites/default/files/publications/publications/p1828_web.pdf

[2] Redmond Ramin Shamshiri, James W. Jones, Kelly R. Thorp, Desa Ahmad, Hasfalina Che Man, and Sima Taheri, "Review of optimum temperature, humidity, and vapour pressure deficit for microclimate evaluation and control in greenhouse cultivation of tomato: a review", Institute of Agrophysics, Polish Academy of Sciences, vol. 32, no. 2, pp. 287-302, 2018. DOI: 10.1515/intag-2017-0005.

[3] H.R. Gislerod, A.R. Selmer-Olsen, L.M. Mortensen, "The effect of air humidity on nutrient uptake of some greenhouse plant," Plant and Soil, Martinus Nijhoff Publishers, vol. 102, no. 2, pp. 193-196, 1987. https://www.jstor.org/stable/42936768

[4] Margit Olle, Ingrid H. Williams, "Physiological disorders in tomato and some methods to avoid them," The Journal of Horticultural Science \& Biotechnology, vol. 92, no. 3, pp. 223-230, 2017. DOI: 10.1080/14620316.2016.1255569.

[5] D. Devi Kala Rathinam, D. Surendran, A. Shilpa, A. Santhiya Grace, J. Sherin, "Modern Agriculture Using Wireless Sensor Network (WSN)," International Conference on Advanced Computing and Communication Systems (ICACCS), March 15-16, 2019. DOI: 10.1109/ICACCS.2019.8728284.

[6] Dattatraya Shinde, Naseem Siddiqui, "IOT Based Environment change Monitoring \& Controlling in Greenhouse using WSN," International Conference on Information, Communication, Engineering and Technology (ICICET), August 29-31, 2018. DOI: 10.1109/ICICET.2018.8533808.

[7] Kefaya Qaddoum, E. L. Hines, D. D. Iliescu, "Yield Prediction for Tomato Greenhouse Using EFuNN," Hindawi Publishing Corporation- ISRN Artificial Intelligence, vol. 2013, no. 2, 9 pages, 2013. DOI: $10.1155 / 2013 / 430986$.

[8] Susanto B. Sulistyo, W.L. Woo, S.S. Dlay, "Ensemble Neural Networks and Image Analysis for On-Site Estimation of Nitrogen Contents in Plants," Proceedings of SAI Intelligent Systems Conference (IntelliSys), 2016, DOI: 10.1007/978-3-319-56991-8-9.

[9] Rahamathunnisa U., Nallakaruppan M.K., Anith. A., Sendhil Kumar K.S., "Vegetable Disease Detection Using K-Means Clustering And Svm," International Conference on Advanced Computing and Communication Systems (ICACCS), March 6-7, 2020, DOI: 10.1109/ICACCS48705.2020.9074434.

[10] Usama Mokhtar, Nashwa El Bendary, Aboul Ella Hassenian, E. Emary, Mahmoud A. Mahmoud, Hesham Hefny, Mohamed F. Tolba, "SVM-Based Detection of Tomato Leaves Diseases," International Conference Intelligent Systems IS'2014, Warsaw, Poland, September 24-26, 2014. DOI: 10.1007/978-3-319-113104_55.

[11] Sanjeev S. Sannakki, Vijay S. Rajpurohit, V.B. Nargund, "SVM-DSD: SVM Based Diagnostic System for the Detection of Pomegranate Leaf Diseases," International Conference on Advances in Computing (ICAdC), 2013, pp. 715-720, DOI: 10.1007/978-81-322-0740-5_85.

[12] Zhao Liqiang, Yin Shouyi, Liu Leibo, Zhang Zhen, Wei Shaojun, “A Crop Monitoring System Based on Wireless Sensor Network," Procedia Environmental Sciences, vol. 11, pp. 558-565, 2011. DOI: 10.1016/j.proenv.2011.12.088.

[13] Xu. Guili, Zhang F., Shah S.G., Ye. Yongquiang, Mao H., "Use of leaf color images to identify nitrogen and potassium deficient tomatoes," Pattern Recognition Letters, vol. 33, no. 11, pp. 1584-1590, 2011. DOI: 10.1016/j.patrec.2011.04.020.

[14] Picon A., Alvarez-Gila A., Seitz M., Ortiz-Barredo A.,Echazarra J., Johannes, "Deep convolutional neural networks for mobile capture device-based crop disease classification in the wild," Computers and Electronics in Agriculture, vol. 161, pp. 280-290. DOI: 10.1016/j.compag.2018.04.002.

[15] Aravind Krishnaswamy Rangarajan, Raja Purushothaman, Aniirudh Ramesh, "Tomato crop disease classification using pre-trained deep learning algorithms," International Conference on Robotics and Smart Manufacturing (RoSMa2018), 2018, pp. 1040-1047. DOI: 10.1016/j.procs.2018.07.070.

[16] Yan Guo, Jin Zhang, Chengxin Yin, Xiaonan Hu, Yo Zou, Zhipeng Xue, Wei Wang, "Plant Disease Identification Based on Deep Learning Algorithm in Smart Farming," Discrete Dynamics in Nature and Society, vol. 2020, no. 7, pp. 1-11, 2020. DOI: 10.1155/2020/2479172. 